\title{
Cardiovascular disease primary prevention failure: A sixth of patients with a QRISK2 score below 10\% were started on statins, despite the guidelines
}

\section{Ferruccio De Lorenzo*}

Consultant Physician in General (I) Medicine \& Cardiovascular Disease Prevention, Cardiovascular Disease Prevention \& Lipid Clinic, Hammersmith Hospital NHS Foundation Trust, London, UK

According to the National Institute for Health and Care Excellence (NICE), GPs should use a 10-year cardiovascular disease (CVD) risk estimate, the QRISK'2 score, to determine whether patients should be started on statins. Sam Finnikin and colleagues [1] from the University of Birmingham examined the records of over 1.4 million patients aged over 40 years, and found that just over $10 \%$ had a recorded CVD risk score and that $15 \%$ had been started on statins since 2012 . However, only about a quarter of these newly-treated patients had a record of a risk assessment in their notes, and of those whose risk scores had been calculated, only a minority had started treatment. Even among high-risk patients, only $35 \%$ had been initiated on a statin. This study shows that there is still potential under treatment of patients at high risk of CVD.

In 2012 NICE recommended GPs to use the QRISK2 score to help decide if patients should start on statins. But only around a quarter of patients who began taking the drugs after 2012 have a QRISK2 score documented in their patient record. Of those who do have a recorded score, only $35 \%$ of those with high score were started on statins.
Copyright: (2017 De Lorenzo F. This is an open-access article distributed under the terms of the Creative Commons Attribution License, which permits unrestricted use, distribution, and reproduction in any medium, provided the original author and source are credited.
When NICE lowered the criteria in 2014, setting the threshold for considering offering statins at a $10 \%$ risk over 10 years rather than $20 \%$, initiation rates fell among patients at high risk but rose among patients at medium risk. A sixth of patients with a QRISK2 score below 10\% were started on statins, despite the guidelines.

The Lipid and cardiovascular risk clinics provide specialist lipidology and cardiovascular risk-factor management for outpatient and inpatient management of patients at cardiovascular risk, and should also start to play a major role in implementing cardiovascular risk assessment-not only in the area of secondary prevention, but also primary prevention services.

Further research needs to be carried out to understand the potential role of Lipid clinics in implementing risk assessment before statin initiation with the collaboration of general practices.

\section{References}

1. Finnikin S, Ryan R, Marshall T (2017) Statin initiations and QRISK2 scoring in UK general practice: a THIN database study. $B r J$ Gen Pract pii: bjgp17X693485. [Crossref]
Correspondence to: Ferruccio De Lorenzo, MD PhD FBIHS, Consultant Physician in General (I) Medicine \& Cardiovascular Disease Prevention, Cardiovascular Disease Prevention \& Lipid Clinic, Hammersmith Hospital NHS Foundation Trust, London, UK, E-mail: fdlx@btinternet.com

Received: October 23, 2017; Accepted: November 06, 2017; Published: November 10, 2017 